\title{
GESTATIONAL TROPHOBLASTIC NEOPLASIA: COMPUTED TOMOGRAPHIC APPEARANCE
}

\author{
Marco A. Amendola, ${ }^{1}$ Mark H. Jaffe, ${ }^{1}$ Beatriz E. Amendola ${ }^{1}$ \\ and JAMES S. HOFFMAN ${ }^{2}$ \\ Departments of ${ }^{1}$ Radiology and, ${ }^{2}$ Obstetrics and Gynecology, University of Michigan Medical Center, \\ Ann Arbor, MI 48109, U.S.A.
}

(Received 4 March 1982; received for publication 17 August 1982)

\begin{abstract}
The authors present the computed tomographic findings in a patient with malignant gestational trophoblastic neoplasia. No similar case has been described on computed tomography. The interesting aspects of the case and a brief review of this clinical entity is discussed.
\end{abstract}

Pelvic computed tomography Malignant gestational trophoblastic neoplasia Theca-lutein cysts Ovary

\section{INTRODUCTION}

Gestational trophoblastic neoplasia (GTN) includes the entities of choriocarcinoma, invasive molar disease and metastatic molar disease [1]. All are sequelae to human pregnancy. Approximately half of all patients who develop malignant GTN have had an antecedent molar gestation, while the remaining half have had other types of antecedent pregnancy, including term delivery, abortion, therapeutic or spontaneous, stillbirth, or even ectopic gestation.

Molar pregnancy is generally suspected in the setting of abnormal bleeding, abnormal uterine growth and absent fetal heart tones. The condition is usually diagnosed by ultrasound and treated by suction curettage. Twenty percent of patients with hydatidiform moles can be expected to develop subsequent malignant sequelae [2]. For this reason it is mandatory to follow these patients with serial levels of beta human chorionic gonadotropin (Beta-HCG). If these levels persist or increase then the patients must be assessed for possible metastatic disease. It has been recently indicated that the risk of GTN in molar pregnancies is significantly increased in pregnancies large for dates with a uterus or more than 20 weeks' gestational size and with theca-lutein cysts greater than $5 \mathrm{~cm}$ in diameter $[2,3]$. The uterine abnormality and the bilateral theca-lutein cysts are well demonstrated in this report of the CT findings in a patient with metastatic molar disease.

\section{CASE REPORT}

A 26-yr old white female, gravida 1 para 1, sought medical advice in September 1980, for abnormal vaginal bleeding. Her last menstrual period had been two months earlier, and she thought she was pregnant. D \& C was performed, and pathologic examination of the curetted specimen revealed grade III of III molar tissue. Serial serum beta-HCG increased markedly in subsequent weeks and a repeat D \& C in November yielded grade III molar tissue. The patient was referred to the University Hospital for staging and initiation of chemotherapy for trophoblastic disease. Physical and pelvic examination disclosed bilateral large ovarian cysts, each about $10-12 \mathrm{~cm}$ in size, suggestive of theca-lutein cysts. The uterus was slightly enlarged to about the size of a 7 week pregnancy, anteverted and smooth with no vaginal disease palpated.

Chest X-rays and polydirectional lung tomograms revealed 2 metastatic nodules. A CT scan of the brain was normal. CT scan of the abdomen did not demonstrate liver metastases. There was no evidence of adenopathy or other intra-abdominal metastases however, bilateral large, septated fluidfilled masses were noted, each just anterior to the iliopsoas and ureter extending to the anterior MI 48109, U.S.A. 

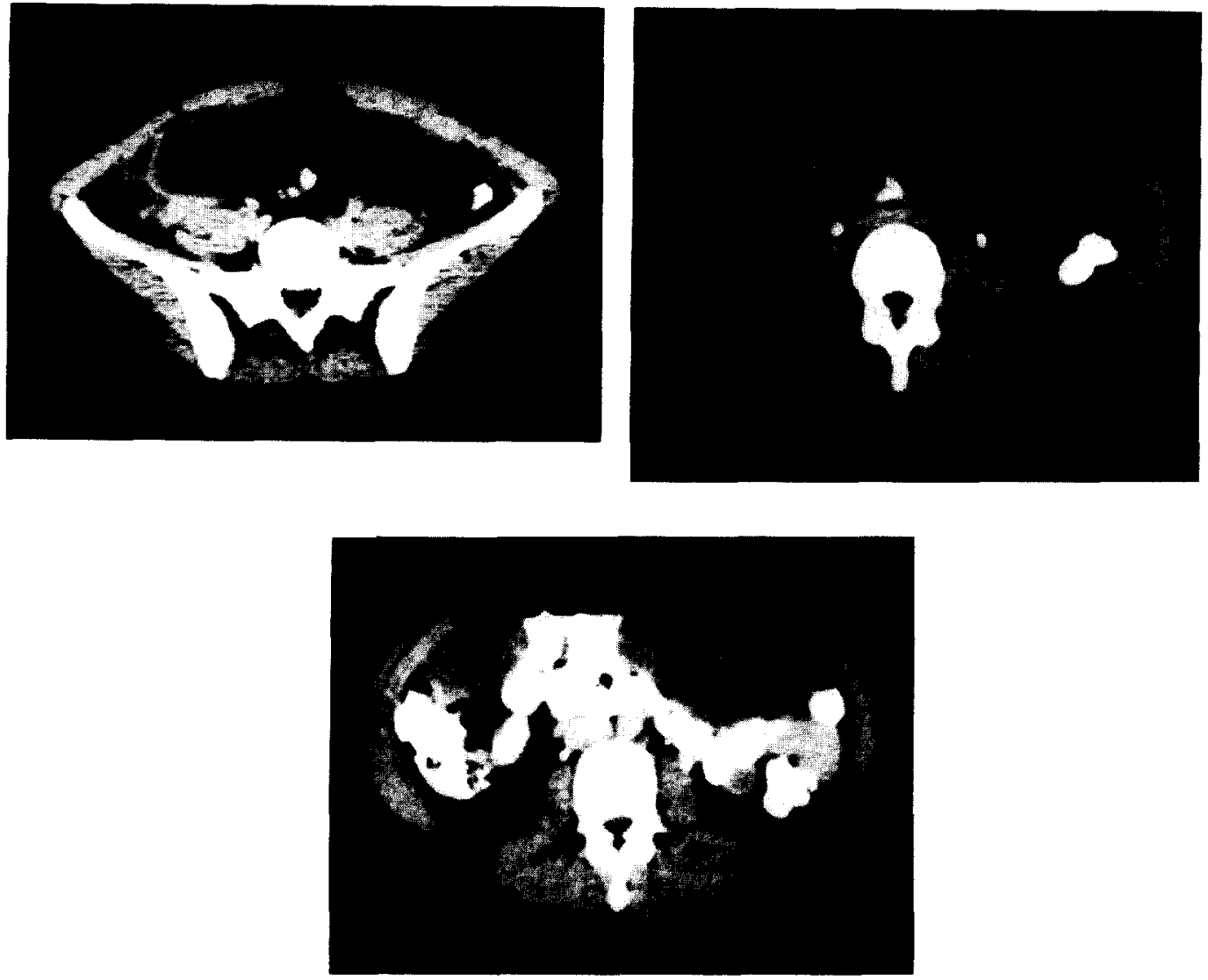

Fig. 1. (A-C) Bilateral theca-lutein cysts: large multiseptated masses of fluid density (cursor marker indicates 13 Hounsfield units) extending upwards from the pelvis (A) into midabdomen (B,C). The margins of the masses are well demarcated with displacement of contrast filled loops of bowel posteriorly and laterally.

abdominal wall and displacing bowel to either side (Fig. 1). The appearance was felt to be consistent with large bilateral theca-lutein cysts of the ovaries. The uterus was enlarged with low attenuation areas (Fig. 2). It measured $10 \mathrm{~cm}$ in both AP and transverse dimensions. Sonography was not performed.

Significant laboratory tests included HCG on $24 \mathrm{~h}$ urine with HCG daily output of between 33,500 and 50,215 units. On the basis of all the above data, the patient was enrolled in a low risk metastatic trophoblastic neoplasia treatment group, (Class IV in the New England Trophoblastic Disease

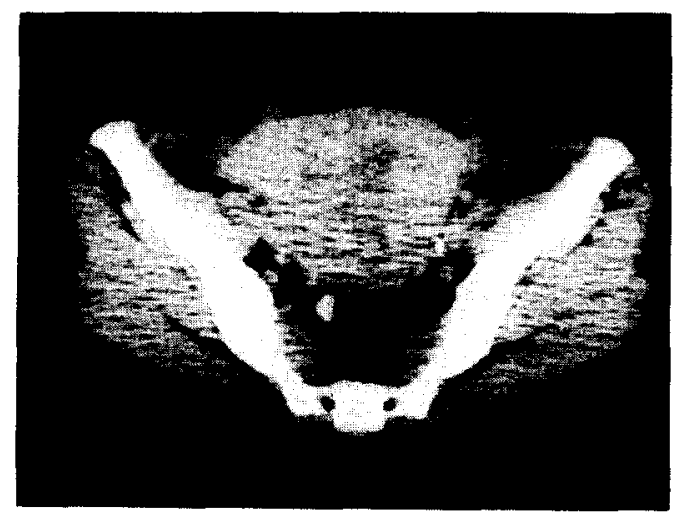

Fig. 2. Enlarged uterus $(10 \times 10 \mathrm{~cm})$ containing areas of low attenuation. Curettage 3 weeks prior had demonstrated grade III molar tissue. 
Center classification), and was started on methotrexate therapy. Four months later and after 9 courses of methotrexate, physical and pelvic examination had returned to normal. Pulmonary nodules had resolved on chest radiographs and beta-HCG levels were not detectable.

\section{DISCUSSION}

The usefulness of ultrasonography for the diagnosis of trophoblastic disease has been well established [4-6]. In our patient CT demonstrated bilateral large multiseptated masses of fluid density closely resembling the classic sonographic appearance of theca-lutein cysts well described by Kobayashi and others $[7,8]$. The cystic masses were well circumscribed and separate from other structures except for displacement of adjacent bowel. A histologic diagnosis cannot be offered by CT and other multiseptated cystic masses might have been included in the differential diagnosis, but the clinical course was most consistent with the CT impression of theca-lutein cysts. CT has recently been reported to be useful in the imaging of tumors of ovarian origin as small as $3 \mathrm{~cm}$ in maximal diameter [9].

Theca-lutein cysts in GTN are due to excessive HCG produced by the molar pregnancy. Patients who have these cysts will have a significantly greater risk of persistent trophoblastic disease [3]. Palpable theca-lutein cysts are present in about $30 \%$ of molar pregnancies; however, the reported incidence of these masses on ultrasound examination has been as high as $50 \%$ [4]. In a recently reported series 5 of 16 patients studied with ultrasonography had cysts of less than $300 \mathrm{~cm}^{3}$ in volume, which were not palpable on physical examination [10].

Recognition of invasive trophoblastic disease on ultrasound depends on the presence of hemorrhagic necrosis within the myometrium, manifested as arcas of irregular hypoechoicity and high amplitude echoes in clusters [8]. However, occasionally, invasive trophoblastic disease can be present without recognizable textural abnormalities of the uterus on ultrasound [8]. Even curettage may not identify residual disease present deep in the myometrium [1]. Although definitive pathological proof was not obtained, the CT appearance of uterine enlargement with irregular areas of low attenuation in our patient, was highly suggestive of invasive trophoblastic disease.

In patients with GTN, metastatic deposits can be found in the gastrointestinal and genitourinary tracts as well as in the liver, lung and brain. Even in this highly responsive disease, cerebral, hepatic and bowel metastases are difficult to control. Detection of trophoblastic disease in these sites places the patient in a high risk category with poor prognosis and demands a much more aggressive therapeutic approach. Cranial CT is the preferred diagnostic modality in the search for metastatic disease to the brain $[1,11]$. Although evaluation of liver involvement by scintigraphy is generally advocated, the high degree of accuracy of CT in detecting hepatic neoplasm [11], as well as other areas of abdominal involvement, may be of benefit in selected patients. Familiarity with the CT appearance we describe may be also helpful in those patients in whom invasive trophoblastic disease is not suspected, i.e. those without antecedent mole and whose preceding pregnancy occurred years previously (or who actually had a recent pregnancy with early unrecognized abortion). In those instances determination of serum beta-HCG level, a highly sensitive and accurate tumor marker. may be obtained to confirm the CT diagnosis.

\section{SUMMARY}

The computed tomographic findings in a patient with gestational trophoblastic neoplasia are presented for the first time. Although computed tomography is not advocated as the modality of choice for imaging of the primary disease, awareness of the CT appearance described may be helpful in cases without clear-cut history pointing towards gestational trophoblastic malignancy. The possible role of computed tomography in the search for distant metastases is discussed.

\section{REFERENCES}

1. P. Disaia and W. T. Creasman, Clinical Gynecologic Oncology, pp. 166-184. Mosby, St Louis, MO (1981).

2. S. L. Curry, C. B. Hammond, L. Tyrey et al., Hydatidiform mole: Diagnosis, management and long term follow-up of 347 patients, Obstet. Gynecol. 45, 1-8 (1975).

3. C. P. Morrow, O. A. Kletzky and P. J. Disaia et al., Clinical and laboratory correlates of molar pregnancy and trophoblastic disease, Am. J. Obstet. Gynecol. 128, 424-430 (1977). 
4. A. M. Baird, D. E. Beckly and F. G. M. Ross, The ultrasound diagnosis of hydatidiform mole, Clin. Radiol. 28, 637-645 (1977).

5. A. C. Fleischer, A. E. James, D. A. Krause and J. Millis, Sonographic patterns in trophoblastic disease, Radiology 126, $215-220$ (1978).

6. M. Kobayashi, Use of diagnostic ultrasound in trophoblastic neoplasm and ovarian tumors, Cancer (Suppl. 1) 38. $441-452$ (1976).

7. M. Kobayashi, Illustrated Manual of Ultrasonography in Obstetrics and Gynecology, pp. 180-213. Igaku-Shoin, Tokyo (1980).

8. A. C. Fleischer, F. H. Boehm and A. E. James, Sonographic evaluation of trophoblastic disease, in The Principles and Practice of Ultrasonography in Obstetric and Gynecology, R. C. Sanders and A. E. James, Eds, pp. 255-262. AppletonCentury-Crofts, New York (1980).

9. M. A. Amendola, J. W. Walsh and B. E. Amendola et al., Computed tomography in the evaluation of carcinoma of the ovary, J. Comput. assist. Tomogr. 5, 169-186 (1981).

10. K. C. Requard and F. A. Mettler, The use of ultrasound in the evaluation of trophoblastic disease and its response to therapy, Radiology 135, 419-422 (1980).

11. E. A. Surwit and C. B. Hammond, Treatment of metastatic trophoblastic disease with poor prognosis, Obstet. Gynecol. 55, 565-570 (1980).

12. J. H. Snow, H. M. Goldstein and S. Wallace, Comparison of scintigraphy, sonography and computed tomography in the evaluation of hepatic neoplasms, Am. J. Roentg. 132, 915-918 (1978).

About the Author--Marco A. Amendola is currently an Associate Professor of Radiology at the University of Michigan. He received his M.D. degree from the School of Medicine, University of Uruguay, Montevideo. His residency in Radiology and fellowship in Computed Tomography were completed at the Albert Einstein Medical Center, Philadelphia, Pennsylvania. He is a Director of Genito-Urinary Radiology, and Co-Director of Abdominal Radiology Division at the University of Michigan Medical Center in Ann Arbor, Michigan.

About the Author-Mark H. JAFFe received his B.A. degree from Yale University in 1970 and his M.D. degree from Washington University in 1974. His residency was in Diagnostic Radiology at Yale-New Haven Hospital and since then he has held faculty positions in Radiology at the University of Chicago and the University of Michigan. He is presently a Director of Gastrointestinal Radiology and Co-Director of the Abdominal Radiology Division at the University of Michigan Medical Center in Ann Arbor, Michigan.

About the Author-BEatriz E. AmEndola received her M.D. degree from the School of Medicine, University of Uruguay, Montevideo, in 1974. She received her training in Radiation Oncology at the Medical College of Virginia, Virginia Commonwealth University. She is presently Assistant Professor of Radiology and Acting Chief of the Radiation Therapy Department at the University of Michigan Medical Center, Ann Arbor, Michigan.

About the Author-James S. Hoffman received his M.D. degree from Albert Einstein School of Medicine, New York in 1976. He subsequently completed his residency in Obstetrics and Gynecology and fellowship in Gynecologic Oncology at the Beth Israel Hospital, Boston, Massachussetts and the University of Michigan, Ann Arbor. He is presently Assistant Professor of Obstetrics and Gynecology at the University of Connecticut. 